\title{
LINEAR-COMPLEXITY EQUALIZED MULTIMUSER RECEIVERS FOR WIDEBAND CDMA IN TIME VARYING CHANNELS
}

\author{
Po-Wei Fu, and Kwang Cheng Chen \\ College of Electrical Engineering National Taiwan University, Taipei, Taiwan, R.O.C. \\ FAX: +886223683824 e-mail: powei@euler.ee.nthu.edu.tw, chenkc@cc.ee.ntu.edu.tw
}

\begin{abstract}
In practical environments, CDMA suffers from multiple access interference, inter-symbol interference, and other time-varying effects from the channel. We therefore derive linear complexity multi-user receivers by adaptive algorithms for nonstationary frequency-selective fading channels. Time-varying tapped-delay-line channel model is adopted. Performances of these schemes are shown more effective.
\end{abstract}

\section{INTRODUCTION}

In a practical CDMA system there are two primary issues should be considered: inter-symbol interference (ISI) and multiple access interference (MAI). Consequently, multi-user communication research about MAI and ISI due to multi-path fading attracts great interests, with the application of wideband CDMA systems for high data rate and multi-rate services. ISI effect likely from a dynamic frequency selective channel should be designed carefully with MAI concerns. Such as the multi-path decorrelating receiver in [7], if it acts in a practical environment with more paths, the decorrelating filter will become a nearly singular matrix due to the orthogonal property between signature waveforms. Moreover, most multi-user communication studies usually assume that different users experience the same channel, especially while adopting equalization approach. However, in a multi-user system, different signals go through different paths and thus with different fading result at the receiver end. [6] treated each channel as an individual linear time invariant system during several symbol periods with a different impulse response. In fact, the channel characteristics are time varying and even nonstationary in mobile communication systems. Thus, we take advantage of a general time varying Tapped-Delay-Line channel model to develop low-complexity multi-user receiver in slow time-varying frequency selective fading environment.

In this case, channel equalization is generally required to mitigate the effect of ISI and also effective to reduce MAI. However, theoretical equalization methods require a priori knowledge of channel parameters, which implies the need of accurate channel estimates to invoke tremendous system complexity. Instead, we proceed without channel identification to derive multi-user receiver structures of linear complexity and implement them by adaptive schemes to adapt the channel variations.

\section{SYSTEM MODEL}

We consider a synchronous CDMA system with $K$ users transmitted over respective frequency-selective fading channels with additive white Gaussian noise (AWGN). The low-pass equivalent transmitted signal for the user $k$ is: $x_{k}(t)=A_{k} \sum_{i=-\infty}^{\infty} b_{k}(i) S_{k}(t-i T)$

where $A_{k}$ and $S_{k}$ are the transmitted amplitude and the signature waveform of the $k$ th user respectively. The data symbols of all users are i.i.d. and with baseband antipodal modulation. $b_{k} \in\{1,-1\} . T$ is the symbol period. The signature waveform of the user $k$ is:

$S_{k}(t)=\sum_{j=0}^{N-1} C_{k}(j) p\left(t-j T_{c}\right) \quad 0 \leq t \leq T$

where $C_{k}$ is the spreading code (signature sequence) of the user $k, \varphi(t)$ is a normalized rectangular chip function with duration $T_{c}$, and $N$ is the procession gain, $T=N T_{c}$. Each user's signal is transmitted through an individual frequencyselective fading channel embedded in AWGN, which is modeled as Tapped-Delay-Line form [4]. This channel model is composed of random coefficients and independent to each other. Assumed that all users are received coherently, then the received signal of the $k$ th user is:

$$
y_{k}(t)=A_{k} \sum_{i=-\infty}^{\infty} \sum_{n=0}^{4-1} \sum_{j=0}^{N-1} b_{k}(i) C_{k}(j) d_{k n}(t) \rho\left(t-\frac{n}{W}-i T-j T_{c}-\tau_{k}\right)
$$

where $W$ is the bandwidth of the transmitted signals $=1 / T c$, $\tau_{k}$ is the transmission delay, $L_{k}=\left\lfloor T_{k m} W\right\rfloor+1$ is the number of channel taps, and $T_{k m}$ denotes the delay spread of the user $k$ 's channel. The time-variant $d_{k n}(t)$ is the $n$th tap coefficient of the user $k^{\prime}$ 's channel at time $t$, which is a complex-valued stochastic process. $L_{k}$ is the number of resolvable paths, which could be set as a constant maximum value $L$ for all user's channels. $d_{k n}(t)$ remains constant during a symbol period. The channels are assumed to suffer from Raylaigh Fading effect that $d_{k n}(t)$ is a complex Gaussian random process. It is reasonable to assume $d_{k n}(t)$ constant during a 
symbol period, especially when the data rate is high. Therefore, the final received signal is:

$\left.n(t)=\sum_{k=1}^{K} A_{k} \sum_{n=0}^{L-1} \sum_{j=0}^{N-1} \sum_{i=-\infty}^{\infty} b_{k}(i) C_{k}(j) d_{k n}(i) d t-(n+i N+j) T_{c}-\tau_{k}\right]+n(t)$

where $n(t)$ is a complex-valued AWGN with zero mean and power spectrum density $N_{0} \cdot \tau_{k}=0$ for synchronous CDMA.

\section{RECEIVER STRUCTURE}

For a signal with bandwidth $W$, the resolution of its multiple paths is approximately equal to $1 / W$. To equalize the unknown composite channel, we use a chip matched filter and then sample the output at chip rate, $\int_{0}^{T_{C}}\{\}_{p}(t) t t$. The output samples construct sufficient statistics. The sampled signal after the $l$ th symbol, $m$ th chip time is:

$$
\begin{aligned}
& r_{m}(l)=\sum_{k=1}^{K} \sum_{i=0}^{M-1} b_{k}(l-i) h_{k m}(l ; i)+v_{m}(l) \\
& h_{k m}(l ; i) \equiv A_{k} \sum_{j=0}^{N-1 L-1} C_{k=0}(j) d_{k n}(l-i) \int_{0}^{T_{c}} \varphi\left[t+(i N+m-j-n) T_{c}\right] \rho(t) d t
\end{aligned}
$$

where $v_{m}(l)$ is from the background noise, and $M$ $=\lceil(L-1) / N\rceil+1$. The output samples during each symbol period are collected as a processing vector form:

$\mathbf{r}(l)=\left[r_{0}(l) r_{1}(l) \cdots r_{N-1}(l)\right]^{r}$.

$=[\mathbf{h}(l, M-1) \mathbf{h}(l, M) \cdots \mathbf{h}(l, 0)] \mathbf{b}(l-M+1) \mathbf{b}(l-M+2) \cdots \mathbf{b}(l)]^{\Gamma}+\mathbf{V}(l)$

Not knowing the channel parameters, to equalize the overall channel for extracting the $l$ th data symbol of the user $k$, we stack the successive $P$ output vectors since the desired signal responds on the $l$ th, $(l+1)$ th, $(l+P-1)$ th symbol periods, where $P=\lceil(L-1) / N\rceil+1=M$. We form our processing vector $\mathbf{R}_{M}(l)$ from $\mathbf{r}(l)$. The processing delay is at least $M T$. The depth of stacking is a tradeoff between the performance and complexity, and here we just adopt the basic complexity for $P=M$. It follows: $\mathbf{R}_{M}(l)=\mathbf{y}_{M}(l)+\mathbf{V}_{M}(l)=\mathbf{H}_{M}(l) \mathbf{B}_{M}(l)+\mathbf{V}_{M}(l)$

$$
=\left[\begin{array}{ccccc}
\mathrm{h}(l, M-1) \mathrm{h}(l, M-2) \cdots \mathrm{h}(l, 0) & 0 & \cdots & 0 \\
0 & \mathrm{~h}(l+1, M-1) \cdots \cdots \mathrm{h}(l+1,0) & 0 \cdots & 0 \\
\vdots & \ddots & \ddots & \ddots & \\
0 & \cdots & 0 & \mathrm{~h}(l+M-1, M-1) & \cdots \mathrm{h}(l+M-1,0)
\end{array}\right]\left[\begin{array}{c}
\mathrm{b}(l-M+1) \\
\mathbf{b}(l-M+2) \\
\vdots \\
\mathbf{b}(l+M-1)
\end{array}\right]+\mathrm{V}_{M}(l)
$$

where $\mathbf{H}_{M}(l), \mathbf{R}_{M}(l)$, and $\mathbf{B}_{M}(l)$ are $M N \times K(M+L-1)$, $M N \times 1$ and $K(M+L-1) \times 1$ matrixes respectively.

Fig. 1 shows the general structure of the receivers. For the user $k$ 's $l$ th symbol detection, the adaptive filter shown in Fig. 2 with $N$ branches and $M$ stages is denoted as a vector $\mathbf{W}_{k}(l)_{M N \times 1}$. There are $N$ branches at the output of the serial-to-parallel device and each branch corresponds to a spreading code position. The finial decision is made as:
$\hat{b}_{k}(l)=\operatorname{sgn}\left\{\mathfrak{R} e\left[\mathbf{W}_{k}(l) \mathbf{R}_{M}(l)\right]\right\}$

In the following, we derive two forms of the linear complexity receivers and evaluate their performance in non-stationary channels.

\section{A. Linear MMSE Receiver}

A typical approach is to use Minimum Mean Squared Error (MSE) criterion, MSE $J(l) \equiv E\left[\left(b_{k}(l)-\tilde{b}_{k}(l)\right)^{2}\right] . J(l)$ and its minimum value are variant with $l$ in non-stationary channels. To minimize $J(l)$ with respect to $\mathbf{W}_{k}(l)$, by the orthogonal principle,

$$
E\left[\mathbf{R}_{M}(l) b_{k}^{*}(l)\right]_{M N \times 1}=E\left[\mathbf{R}_{M}(l) \mathbf{R}_{M}^{H}(l) \cdot \mathbf{w}_{k}^{H}(l)\right]_{M N \times 1}
$$

The optimum coefficients of the filter for user $k$ are time varying due to the stochastic non-stationary characteristics of the composite channel. In case the channel is time varying but stochastically stationary or even fixed, with the stationary data symbols and the cyclic stationary spreading codes, $\mathbf{R}_{M}(l)$ is statistically stationary and then the index $l$ of (3.5) disappears as in a traditional Wiener-Hoff equation, i.e. $\mathbf{w}_{k}^{H}=E\left[\mathbf{R}_{M}(l) \mathbf{R}_{M}^{H}(l)\right]^{-1} E\left[\mathbf{R}_{M}(l) b_{k}^{*}(l)\right]$.

If the composite channel is non-stationary, we should solve (3.5) to derive the optimum solution of the $l$ th period, assumed that we could know the relationship between the ensemble average $E\left[\mathbf{R}_{M}(l) \mathbf{R}_{M}^{H}(l) \cdot \mathbf{W}_{k}^{H}(l)\right]$ and the optimum $\mathbf{W}_{k}(l)$ and the cross-correlation $E\left[\mathbf{R}_{M}(l) \boldsymbol{B}_{k}^{*}(l)\right]$. Unfortunately, it is difficult to separate $\mathbf{W}_{k}(l)$ from the ensemble average term unless we constraint the filer coefficients to be fixed values (i.e. uncorrelated with $\left.\mathbf{R}_{M}(l) \mathbf{R}_{M}^{H}(l)\right)$ for simplicity. However, for wideband CDMA systems, it easily achieves a "locally stationary" condition. There are $M+L-1$ symbols of each user involved in the processing vector $\mathbf{R}_{M}(l)$, so if the channel is stationary at least for $M+L-1$ symbol periods, an fixed optimum solution exits for each processing vector based on its local statistics. If the processing vector is locally stationary,

$$
\begin{aligned}
& E\left[\mathbf{R}_{M}(l) b_{k}^{*}(l)\right]=E\left[\mathbf{R}_{M}\left(l \mathbf{R}_{M}^{H}(l)\right] \mathbf{w}_{k}^{H}(l), \mathbf{W}_{k}^{H}(l)=\mathfrak{R}^{-1}(l) \mathfrak{R}_{k}(l) .\right. \\
& \mathfrak{R}(l) \equiv E\left[\mathbf{R}_{M}(l) \mathbf{R}_{M}^{H}(l)\right] \text { and } \mathfrak{R}_{k}(l) \equiv E\left[\mathbf{R}_{M}(l) b_{k}^{*}(l)\right]
\end{aligned}
$$

The auto-correlation $\Re(l)$ and cross-correlation $\Re_{k}(l)$ are random, and thus $\mathbf{W}_{k}(l)$ is a random process. But with certain specific $l$, it is fixed given the statistics of the processing vector. Hence, within a locally stationary period, the receiver should search an optimum solution for that period and then to track the optimum solutions over successive periods. 
To derive the theoretically achievable MMSE, denote the optimum error between the desired symbol and the optimum estimate as $\breve{e}(l)$, and the optimum coefficient as $\overline{\mathbf{w}}_{k}(l)$. $b_{k}(l)=\check{e}(l)+\breve{\mathbf{W}}_{k}(l) \mathbf{R}_{M}(l)$

Taking mean squared operation, under the locally stationary condition, $J_{\min }(l)=\sigma_{b_{k}}^{2}(l)-\breve{\mathbf{W}}_{k}(l) \Re(l) \breve{\mathbf{W}}_{k}^{H}(l)$

$=\sigma_{b_{k}}^{2}(l)-\mathfrak{R}_{k}^{H}(l)\left(\mathfrak{R}(l)^{-1}\right)^{H} \mathfrak{R}_{k}(l)$

From (3.9), it shows that the achievable MMSE depends on the statistical characteristics of $\mathfrak{R}(l)$ and $\mathfrak{R}_{k}(l)$ for each locally stationary period.

\section{B. Decision Feedback Equalization Receiver}

Another typical thinking is the decision feedback equalization (DFE) filter for each user. Its feedforward part structure is identical to the linear filter described earlier and the feedback part has a tapped-delay structure with tap space $T$ (Fig.3). The processing vector $\mathbf{R}_{M}(l)$ is still used as the input observation of the feed forward part. Since at most $M=\lceil(L-1) / N\rceil-1$ preceding symbols of all users involve $\mathbf{R}_{M}(l)$, we take $M$ previously decisions as the input of the feedback part. Hence, the processing vector

$\mathbf{R}_{B}=\left[\mathbf{R}_{M}(l) \mathbf{b}_{k}(l-1) \cdot \cdot \mathbf{b}_{k}(l-M+1)\right]^{T}, \mathbf{W}_{k}(l)=\left[\mathbf{W}^{f}(l) \mathbf{W}^{b}(l)\right]$

where $\mathbf{W}^{f}(l)$ and $\mathbf{W}^{b}(l)$ denote the tap coefficients of the feed forward and the feedback filter respectively. The estimate of the lth symbol of the $k t h$ user after the DFE is: $\tilde{b}_{k}(l)=\mathbf{W}_{k}(l) \mathbf{R}_{B}(l)$

We use MMSE criterion. Based on the assumption that the previous symbols are detected correctly, it follows that

$$
E\left[\mathbf{R}_{B}(l) \boldsymbol{b}_{k}^{*}(l)\right]=E\left[\mathbf{R}_{B}(l) \mathbf{R}_{B}^{H}(l) \mathbf{W}_{k}^{H}(l)\right]
$$

It is similar to that of the linear MMSE filter case. The optimum $\mathbf{W}_{k}(l)$ is the solution of (3.11). Also, if the processing vector falls in a locally stationary period,

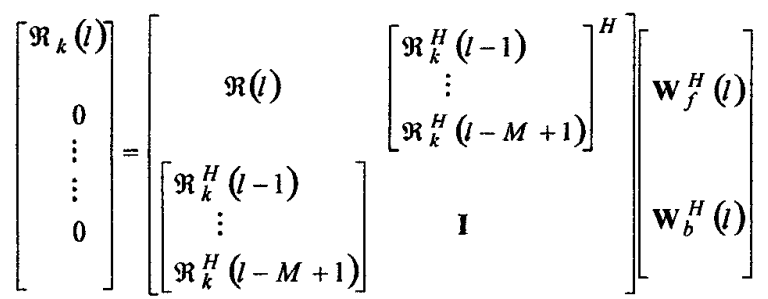

The optimum coefficient

$$
\begin{aligned}
& \mathbf{W}_{f}(l)=\mathfrak{R}_{k}^{H}(l)\left(\mathfrak{R}^{H}(l)-\mathbf{G}(l, M) \mathbf{G}^{H}(l, M)\right)^{-1} \\
& \mathbf{W}_{b}(l)=-\mathbf{W}_{f}(l) \mathbf{G}^{H}(l, M)
\end{aligned}
$$

where $\mathbf{G}(l, M)=\left[\mathfrak{R}_{k}^{H}(l-1) \cdots \mathfrak{R}_{k}^{H}(l-M+1)\right]^{H}$. Therefore, if
$\Re_{k}(l)$ and $\mathfrak{R}(l)$ are known, we can derive the optimum DFE coefficients from (3.13). We make use of Kalman filtering and simpler algorithms as LMS and RLS to adaptively track the optimum solutions over successive stationary periods.

\section{Kalman Filtering}

By assuming a non-linear state transition for optimum solutions and by Taylor expansion of the non-linear function, Extended Kalman filter [2] is adopted. Consider the coefficient vector $\mathbf{W}_{k}(l)$ as the state of the receiver of the user $k$ for $l$ th symbol and then assume the state varies as the following model:

$\mathbf{W}_{k}(l+1)=F\left(l ; \mathbf{W}_{k}(l)\right)+\mathbf{V}_{\mathbf{l}}(l)$ $F\left(l ; \mathbf{W}_{k}(l)\right)$ denotes the transition matrix function which is a functional of the discrete time index $l$ and the present state $\mathbf{w}_{k}(l)$, and $\mathbf{v}_{1}(l)$ is a zero-mean white noise vector with correlation matrix $\mathbf{Q}_{1}(l)$. The measurement equation is modeled as:

$b_{k}(l)=\mathbf{W}_{k}(l) \mathbf{R}_{M}(l)+V_{2}(l)$

$V_{2}(l)$ is a zero-mean white noise process with correlation function $Q_{2}(l)$ and uncorrelated with $\mathrm{V}_{1}(l)$. To make use of the standard Kalman filtering algorithm, linearize the nonlinear functional with respect to $\mathbf{W}_{k}(l)$ around the optimum filtering coefficients $\breve{\mathbf{W}}_{k}(l)$ by Taylor expansion. $F\left(l ; \mathbf{W}_{k}(l)\right)$

$=F^{\prime}\left(l ; \mathbf{W}_{k}(l)\right) \mathbf{W}_{k}(l)+F\left(l ; \breve{\mathbf{W}}_{k}(l)\right)-F^{\prime}\left(l ; \mathbf{W}_{k}(l)\right) \breve{\mathbf{W}}_{k}(l)+\mathbf{\alpha}(2)$

where $F^{\prime}\left(l ; \mathbf{W}_{k}(l)\right) \equiv \frac{\partial F\left(l ; \mathbf{W}_{k}(l)\right)}{\partial \mathbf{W}_{k}(l)}$ and $\mathbf{O}(2)$ denotes those terms with order higher than one dropped for linearization. Take (3.16) into (3.14), we get the follows: $\mathbf{W}_{k}(l+1)=F^{\prime}\left(l ; \mathbf{w}_{k}(l)\right) \mathbf{w}_{k}(l)+\mathbf{D}(l)+\mathbf{V}_{1}(l)$

where $\mathbf{D}(l) \equiv F\left(l ; \overline{\mathbf{W}}_{k}(l)\right)-F^{\prime}\left(l ; \mathbf{W}_{k}(l)\right) \overline{\mathbf{W}}_{k}(l)$ and all the entries in $\mathbf{D}(l)$ are known at time $l$. With (3.15), as long as $F\left(l ; \mathbf{W}_{k}(l)\right), \mathbf{Q}_{1}(l)$ and $Q_{2}(l)$ are known, the recursive iterations for deriving the MMSE solution proceed as follows: For $l=1,2,3 \ldots$.

$\hat{\mathbf{w}}_{k}(0)=E\left[\mathbf{W}_{k}(1)\right]$

$\mathbf{K}(1,0)=E\left[\left(\mathbf{W}_{k}(1)-E\left[\mathbf{W}_{k}(1)\right]\left(\mathbf{w}_{k}(1)-E\left[\mathbf{w}_{k}(1)\right]\right)\right]\right.$

$\mathbf{G}(l)=\mathbf{K}(l, l-1) \mathbf{R}_{M}(l)\left[\mathbf{R}_{M}^{H}(l) \mathbf{K}(l, l-1) \mathbf{R}(l)+\mathbf{Q}_{2}(l)\right]^{-1}$

$\alpha(l)=b_{k}^{*}(l)-\mathbf{R}_{M}^{H}(l) \hat{\mathbf{W}}_{M}^{H}(l)$

$\breve{\mathbf{W}}_{k}^{H}(l)=\widehat{\mathbf{W}}_{k}^{H}(l-1)+\mathbf{G}(l) \alpha(l)$

$\widehat{\mathbf{W}}_{k}^{H}(l+1)=F\left(l ; \overline{\mathbf{W}}_{k}(l)\right)$

$\mathbf{K}(l)=\left[\mathbf{I}-\mathbf{G}(l) \mathbf{R}_{M}^{H}(l)\right] \mathbf{K}(l, l-1)$

$\mathbf{K}(l+1, l)=F^{\prime}\left(l ; \check{\mathbf{w}}_{k}(l)\right) \mathbf{K}(l) \boldsymbol{F}^{\prime} H\left(l ; \check{\mathbf{w}}_{k}(l)\right)+\mathbf{Q}_{1}(l)$ 
Find $F\left(l ; \mathbf{W}_{k}(l)\right), \mathbf{Q}_{1}(l)$ and $Q_{2}(l)$ from the channel model and the relationship of two consecutive MMSE solutions, and apply them for each concerned environment.

\section{Adaptive Implemention}

The adaptive algorithm used in non-stationary environments needs both good convergence and tracking property. For our several proposed linear receivers, we use the two most widely adopted adaptive algorithms to implement them, the LMS and RLS algorithms. LMS is known for its simple complexity and its robustness due to model independence. RLS could be view as a special case of Kalman filter [5], which is known for its tracking ability.

\section{Least Mean Square Algorithm}

No matter the channel is stationary or not, use the instantaneous estimates to replace the ensemble expectations: $\mathbf{W}_{k}(l+1)=\mathbf{W}_{k}(l)+\mu\left(b_{k}(l)-\tilde{b}_{k}(l)\right) \mathbf{R}_{M}^{H}(l)$

A helpful working rule for choosing $\mu$ follows [2]. The current decision $\hat{b}_{k}(l)$ at the output of the receiver is used to replace $b_{k}(l)$ in (3.19) for decision directed mode. Note: The notation, $\mathbf{R}_{M}(l)$, in (3.19) is replaced by $\mathbf{R}_{B}(l)$ for DFE.

\section{Recursive Least Square Algorithm}

The optimum solution in (3.5) and (3.11) involve the estimation of ensemble averages. Minimize the time-average weighted squared error by Least-Squared method [2] in place of the ensemble expectations to derive the optimum solution. The cost

function is: $\tilde{J}(l)=\sum_{i=0}^{i=l} \lambda^{i-l}\left(b_{k}(l)-\tilde{b}_{k}(l)^{2}\right)$

$\lambda$ is the forgetting factor, which is a positive constant close to 1 . Getting rid of the ensemble averages, the algorithm is used with for stationary and non-stationary cases. By matrix inversion lemma, the RLS algorithm is as follows: For $l=1,2 \cdots$ $\xi(l)=b_{k}(l)-\mathbf{W}_{k}(l-1) \mathbf{R}_{M}(l)$

$\mathbf{K}(l)=\left(\lambda^{-1} \mathbf{P}(l-1) \mathbf{R}_{M}(L)\right) /\left(1+\lambda^{-1} \mathbf{R}_{M}^{H}(l) \mathbf{P}(l-1) \mathbf{R}_{M}(l)\right.$ $\xi(l)=b_{k}(l)-\mathbf{W}_{k}(l-1) \mathbf{R}_{M}(l)$

$\left.\mathbf{w}_{k}^{H}(l)=\mathbf{W}_{k}^{H}(l-1)+\mathbf{K}(l)\right)^{*}(l)$

$\mathbf{P}(l)=\lambda^{-1} \mathbf{P}(l-1)-\lambda^{-1} \mathbf{K}(l) \mathbf{R}{ }_{M}^{H} \mathbf{P}(l-1)$

$\tilde{b}_{k}(l)=\mathbf{W}_{k}(l) \mathbf{R}_{M}(l)$

$\mathbf{W}_{k}^{H}(0)=[0], \quad \mathbf{P}(0)=\delta^{-1} \mathbf{I}, \delta$ is a positive small value. The

current decision $\hat{b}_{k}(l)$ at the output of the receiver is used to replace $b_{k}(l)$ in (3.21) in decision directed mode.

\section{NUMERICAL SIMULATION}

In this section, we present the simulation results of the proposed LMMSE receiver, and DFE receiver with both LMS and RLS Algorithm. There are $K=5$ users in the processing gain $N=16$ CDMA system. 16-length Hadamard Walsh codes are used for our simulation. Independent equally probable binary $\{1,-1\}$ sequences are generated for all users as the data symbols. We assume $A_{k}=1$ for all $k$. For the simulated fading characteristics of the channels, each channel is generated separately by referring the impulse response model of [9]. Assumed the carrier frequency is $2 \mathrm{GHz}$, the transmitted bandwidth is $1.25 \mathrm{MHz}$ and the relative moving velocity between the transmitter and receiver is $60 \mathrm{~km} / \mathrm{hr}$. Thus, the maximum Doppler frequency is $111.11 \mathrm{~Hz}$. We use the first order AR (Gaussian Markov) model to simulate the variation of each tap coefficient $d_{k n}(i)$. Assume that the tap coefficients are invariant during a symbol period, and

$d_{k n}(l+1)=\rho d_{k n}(l)+v_{d}(l)$

where $v_{d}(l)$ is a zero mean complex Gaussian driving noise. $\rho=0.999$ is a good approximation around several thousands symbol periods. The number of resolvable paths $L=26$, and the number of the stacked processing vectors $M=3$. Fig. 4 shows the convergence and tracking performance of all adaptive receivers over 100 independent runs at $E b / N_{0}=$ $20 \mathrm{~dB}$ and initially the training sequence with 100 data symbols was used. $E_{b}$ denotes the transmitted symbol energy. We observed successful tracking to thousand decision-directed operations.

We simulated the BER of each proposed adaptive receiver with respective to different SNR for 1200 data symbols where the front 100 symbols are used in training mode. The conventional LMMSE receiver, decorrelating receiver [3] and the adaptive DFE receivers in [1] and [7] are simulated, shown in Fig. 5. From Fig. 5 and Fig. 6, the DFE receiver with RLS adaptive algorithm performs best. The behaviors of these receivers are affected mostly by interference but noise.

\section{CONCLUSION}

Based on the approach of equalization, we proposed multiuser receiver structures of linear-complexity for wideband CDMA in time-varying frequency-selective fading channels in this paper and demonstrated their superior performance.

\section{REFERENCES}

(1) Majeed Abdulrahman, etc., "Decision Feedback Equalization for CDMA in Indoor Wireless Communications," IEEE JSAC Vol. 12, No. 4, pp.698 706, May 1994.

(2) Simon Haykin, "Adaptive Filter Theory," Third Edition, Prentice Hall, 1996. 
(3) R. Lupas and S. Verdu, "Linear Multiuser Detectors for synchronous code-division multiple-access channels," IEEE Trans. Comm., Vol. 35, pp. 123 136, Jan. 1989.

(4) John G. Proakis, "Digital Communications," third edition, MCGraw Hill, 1995.

(5) A. H. Sayed, and T. Kailath, "A State-space Approach to Adaptive RLS Filtering," IEEE Signal Processing Mag., Vol. 11, pp. 18 60, 1994.

(6) X. Wang, and H.V. Poor, "Blind Equalization and Multiuser Detection in Dispersive CDMA Channels", IEEE Trans. On Comm., Vol. 46, No. 1, pp. 91 103, Jan. 1998.

(7) Zhenhua Xie, etc. "A Family of Suboptimum Detectors for Coherent Multiuser Communications," IEEE JSAC, Vol. 8, pp. 683 690, May 1990.

(8) Z. Zvonar and D. Brady, "Suboptimum Multimuser Detector for Synchronous CDMA Frequency-Selective Rayleigh Fading Channels," Proceedings of Globe-com, pp. 82-85, 1992.

(9) UMTS 30.03 version 3.2.0, TR 101112 V3.2.0 (1998-04)

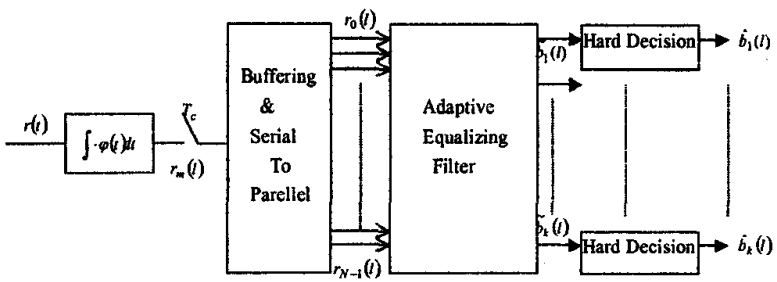

Fig. 1. The structure of the linear equalized receiver

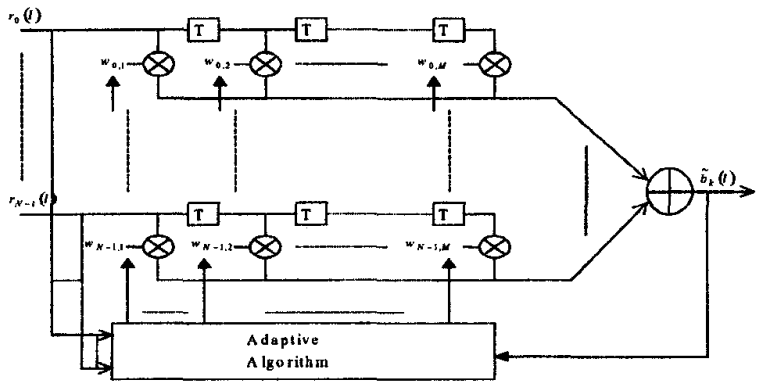

Fig.2.The structure of the $k$ th branch in Adaptive Equalizing Filter device

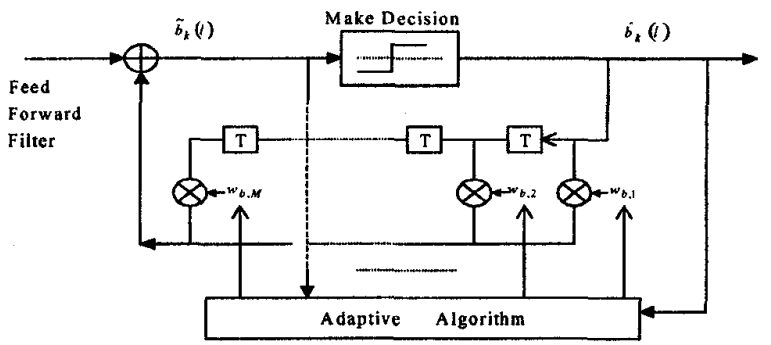

Fig.3. The feed-back filter of the DFE for user $k$
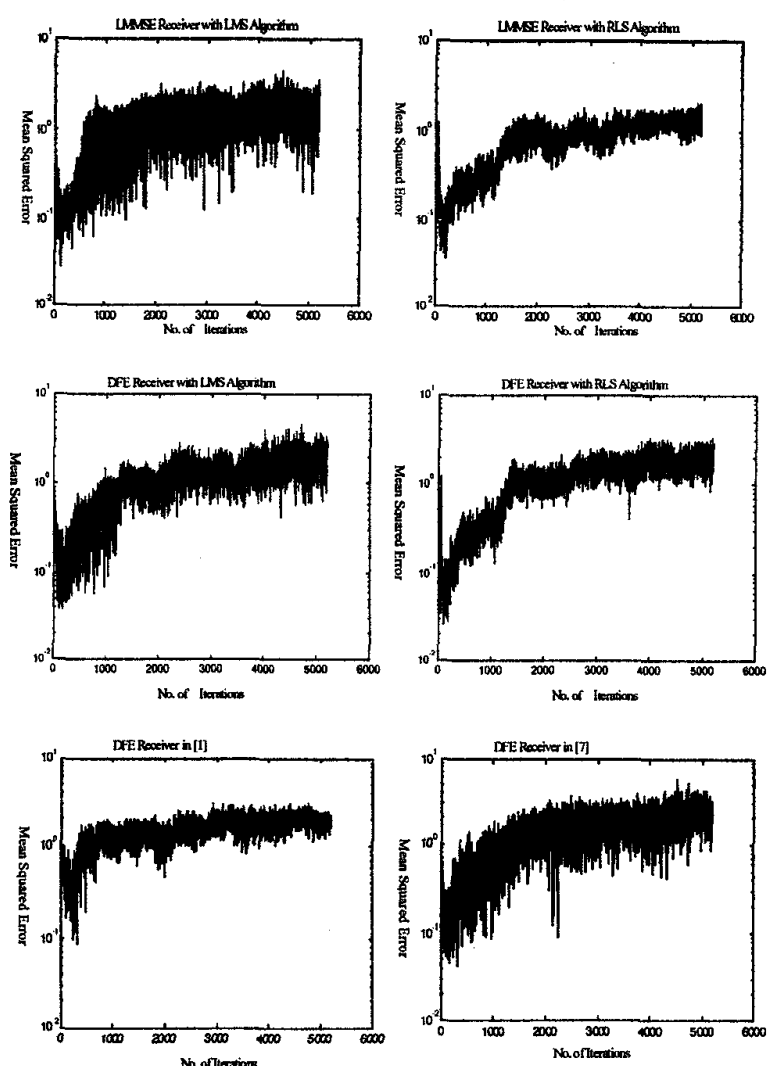

Fig 4 The performance of convergence and tracking

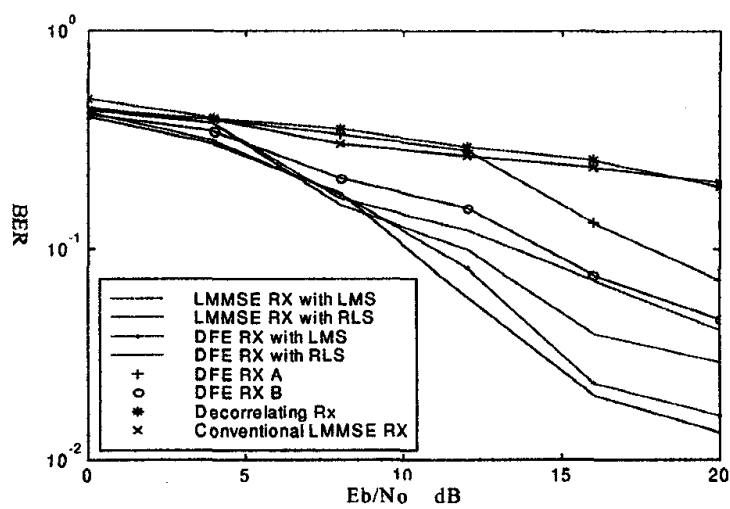

Fig. 5 B it error rate of the four proposed receivers, DFE Fig. 5 Bit error rate of the four proposed receivers, DFE receiver $A[1], D F E$ receiver $B$ [7],
and conventional LMMSE receiver

Acknowledgement: This research was supported by the National Science Council, Taiwan, R.O.C., under the contract NSC-88-2219-E-002-002. 